\title{
Efficiently Mining Approximate Models of Associations in Evolving Databases
}

\author{
Adriano Veloso ${ }^{1}$, Bruno Gusmão ${ }^{1}$, Wagner Meira Jr. ${ }^{1}$, Marcio Carvalho ${ }^{1}$, \\ Srini Parthasarathy ${ }^{2}$, and Mohammed Zaki ${ }^{3}$ \\ ${ }^{1}$ Computer Science Department, Universidade Federal de Minas Gerais, Brazil \\ \{adrianov, gusmao, meira, mlbc\}@dcc.ufmg.br \\ 2 Department of Computer and Information Science, The Ohio-State University, USA \\ srini@cis.ohio-state.edu \\ 3 Computer Science Department, Rensselaer Polytechnic Institute, USA \\ zaki@cs.rpi.edu
}

\begin{abstract}
Much of the existing work in machine learning and data mining has relied on devising efficient techniques to build accurate models from the data. Research on how the accuracy of a model changes as a function of dynamic updates to the databases is very limited. In this work we show that extracting this information: knowing which aspects of the model are changing; and how they are changing as a function of data updates; can be very effective for interactive data mining purposes (where response time is often more important than model quality as long as model quality is not too far off the best (exact) model.

In this paper we consider the problem of generating approximate models within the context of association mining, a key data mining task. We propose a new approach to incrementally generate approximate models of associations in evolving databases. Our approach is able to detect how patterns evolve over time (an interesting result in its own right), and uses this information in generating approximate models with high accuracy at a fraction of the cost (of generating the exact model). Extensive experimental evaluation on real databases demonstrates the effectiveness and advantages of the proposed approach.
\end{abstract}

\section{Introduction}

One of the main characteristics of the digital information era is the ability to store huge amounts of data. However, extracting knowledge, often referred to as data mining, from such data efficiently poses several important challenges. First, the volume of data operated on is typically very large, and the tasks involved are inherently I/O intensive. Second, the computational demands are quite high. Third, many of these datasets are dynamic (E-commerce databases, Web-based applications), in the sense that they are constantly being updated (evolving datasets).

Researchers have evaluated data stratification mechanisms such as sampling to handle the first problem and memory efficient and parallel computing techniques to handle the second problem. Simply re-executing the algorithms to 
handle the third problem results in excessive wastage of computational resources and often does not meet the stringent interactive response times required by the data miner. In these cases, it may not be possible to mine the entire database over and over again. This has motivated the design of incremental algorithms, i.e., algorithms that are capable of updating the frequent itemsets, and thus its associations, by taking into account just the transactions recorded since the last mine operation. In this paper, we propose an approximate incremental algorithm to mine association rules that advances the state-of-the-art in this area.

Association mining is a key data mining task. It is used most often for market basket data analysis, but more recently it has also been used in such far-reaching domains as bioinformatics [7], text mining [14] and scientific computing [9]. Previous research efforts have produced many efficient sequential algorithms $[6,1,8,18,19]$, several parallel algorithms[20,13,3], and a few incremental algorithms for determining associations $[16,15,2,4]$.

The majority of the incremental algorithms studied employ specific data structures to maintain the information previously mined so that it can be augmented by the updates. These techniques are designed to produce exact results, as would be produced by an algorithm running on the entire original database. However, if response time is paramount, these algorithms may still be unacceptable. In this case, it is needed is a way to efficiently estimate the association parameters (support, confidence) without actually computing them and thus saving on both computational and I/O time. Our approach relies on extracting historical trends associated with each itemset and using them to estimate these parameters. For instance, if an itemset support is roughly constant across time, it may not be necessary to compute its exact frequency value. An approximate value may have the same effect. On the other hand, if an itemset shows a consistent increase or decrease trend, its support may be estimated as a function of the number of updates after the last exact count number and the slope associated with the trend.

The main contributions of this paper can be summarized as follows:

- We propose an approximate incremental algorithm, WAVE, for mining association rules, based on trends of itemset frequency value changes.

- We evaluate the above algorithm based on the quality of its estimates (i.e., how close they are from to the exact model) and its performance (when compared against a state-of-the-art incremental algorithm) when mining several real datasets.

We begin by formally presenting the problem of finding association rules in the next section. In Section 3 we present our approach for mining approximate models of associations. The effectiveness of our approach is experimentally analyzed in Section 4. Finally, in Section 5 we conclude our work and present directions for future work. 


\section{Problem Description and Related Work}

\subsection{Association Mining Problem}

The association mining task can be stated as follows: Let $\mathcal{I}=\{1,2, \ldots, n\}$ be a set of $n$ distinct attributes, also called items, and let $\mathcal{D}$ be the input database. Typically $\mathcal{D}$ is arranged as a set of transactions, where each transaction $T$ has a unique identifier TID and contains a set of items such that $T \subseteq I$. A set of items $X \subseteq \mathcal{I}$ is called an itemset. For an itemset $X$, we denote its corresponding tidlist as the set of all TIDs that contain $X$ as a subset. The support of an itemset $X$, denoted $\sigma(X)$, is the percentage of transactions in $\mathcal{D}$ in which $X$ occurs as a subset. An itemset is frequent if its support $\sigma(X) \geq$ minsup, where minsup is a user-specified minimum support threshold.

An association rule is an expression $A \stackrel{p}{\rightarrow} B$, where $A$ and $B$ are itemsets. The support of the rule is $\sigma(A \cup B)$ (i.e., the joint probability of a transaction containing both $A$ and $B)$, and the confidence $p=\sigma(A \cup B) / \sigma(A)$ (i.e., the conditional probability that a transaction contains $B$, given that it contains $A$ ). A rule is frequent if the itemset $A \cup B$ is frequent. A rule is confident if $p \geq$ minconf, where minconf is a user-specified minimum confidence threshold.

Finding frequent itemsets is computationally and I/O intensive. Let $|\mathcal{I}|=m$ be the number of items. The search space for enumeration of all frequent itemsets is $2^{m}$, which is exponential in $m$. This high computational cost may be acceptable when the database is static, but not in domains with evolving data, since the itemset enumeration process will be frequently repeated. In this paper we only deal with how to efficiently mine frequent itemsets in evolving databases.

\subsection{Related Work}

There has been a lot of research in developing efficient algorithms for mining frequent itemsets. A general survey of these algorithms can be found in [17]. Most of these algorithms enumerate all frequent itemsets. There also exist methods which only generate frequent closed itemsets [18] and maximal frequent itemsets [6]. While these methods generate a reduced number of itemsets, they still need to mine the entire database in order to generate the set of valid associations, therefore these methods are not efficient in mining evolving databases.

Some recent effort has been devoted to the problem of incrementally mine frequent itemsets $[10,15,16,2,4,5,12]$. An important subproblem is to determine how often to update the current model. While some algorithms update the model after a fixed number of new transactions $[16,15,2,4]$, the DELI algorithm, proposed by Lee and Cheung [10], uses statistical sampling methods to determine when the current model is outdated. A similar approach proposed by Ganti et al (DEMON [5]) monitors changes in the data stream to determine when to update. An efficient incremental algorithm, called ULI, was proposed by Thomas [15] et al. ULI strives to reduce the $\mathrm{I} / \mathrm{O}$ requirements for updating the set of frequent itemsets by maintaining the previous frequent itemsets and the negative border [11] along with their support counts. The whole database is scanned just 
once, but the incremental database must be scanned as many times as the size of the longest frequent itemset.

The proposed algorithm, WAVE, is different from the above approaches in several ways. First, while these approaches need to perform $O(n)$ database scans ( $n$ is the size of the largest frequent itemset), WAVE requires only one scan on the incremental database and only a partial scan on the original database. Second, WAVE supports selective updates, that is, instead of determining when to update the whole set of frequent itemsets, WAVE identifies specifically which itemsets need to be updated and then updates only those itemsets. Finally, because WAVE employs simple estimation procedures it has the ability to improve the prediction accuracy while maintaining the update costs very small. The combination of incremental techniques and on-the-fly data stream analysis makes WAVE an efficient algorithm for mining frequent itemsets and associations in evolving, and potentially streaming databases.

\section{The ZigZag and Wave Algorithms}

In previous work [16] we presented the ZIGZAG algorithm, a method which efficiently updates the set of frequent itemsets in evolving databases. ZIGZAGis based on maintaining maximal frequent itemsets (and associated supports of all frequent itemset subsets) across database updates. On an update, the maximal frequent itemsets are updated by a backtracking search approach, which is guided by the results of the previous mining iteration. In response a user query ZIGZAG uses the upto-date maximal frequent itemsets ${ }^{1}$ to construct the lattice of frequent itemsets in the database. As shown in[16] this approach of maintaining and tracking maximal itemsets across database updates, results in significant $\mathrm{I} / \mathrm{O}$ and computational savings when compared with other state-of-the-art incremental approaches.

WAVE is an extension to ZIGZAG. WAVE eessentially maintains the same data structure but adds the capability to determine when and how to update the maintained information. It relies on its ability to detect trends and estimate itemset frequency behavior as a function of updates. If an itemset can be well estimated, the exact frequency is not computed, otherwise it will be computed. In comparison to ZIGZAG, WAVE can significantly reduce the computation required to process an update but this reduction comes at some cost to accuracy (since we often estimate rather than compute frequencies). Contrasting to other incremental approaches $[15,2,4,5]$ which generally monitor changes in the database to detect the best moment to update the entire set of itemsets, we choose instead to perform selective updates, that is, the support of every single itemset is completely updated only when we cannot compute a good estimate of its frequency.

Figure 1 depicts a real example that motivates our selective approach. This figure shows the correlation of two sets of popular itemsets. These popular itemsets are ranked by support (i.e., popularity ranking) and their relative positions

\footnotetext{
${ }^{1}$ The maximal frequent itemsets solely determine all frequent itemsets
} 
are compared. When the set of popular itemsets is totally accurate, all the popular itemsets are in the correct position. From Figure 1 we can see a comparison of a totally accurate set of popular itemsets and a ranked set of itemsets which is becoming outdated as the database evolves. As we can see in this figure, although there were significant changes in the support of some popular itemsets, there are also a large number of popular itemsets which remain accurate (i.e., in the correct position) and do not need to be updated, and also a large number of popular itemsets which had evolved in a systematic way, following some type of trend. Our method relies on accurately identifying and categorizing such itemsets. We describe these categories next:

Invariant: The support of the itemset does not change significantly over time (i.e., it varies within a predefined threshold) as we add new transactions. This itemset is stable, and therefore, it need not be updated.

Predictable: It is possible to estimate the support of the itemset within a tolerance. This itemset presents a trend, that is, its support increases or decreases in a systematic way over time.

Unpredictable: It is not possible, given a set of approximation tools, to obtain a good estimate of the itemset support. Note, that it is desirable to have few unpredictable itemsets as these are the ones that cannot be estimated.
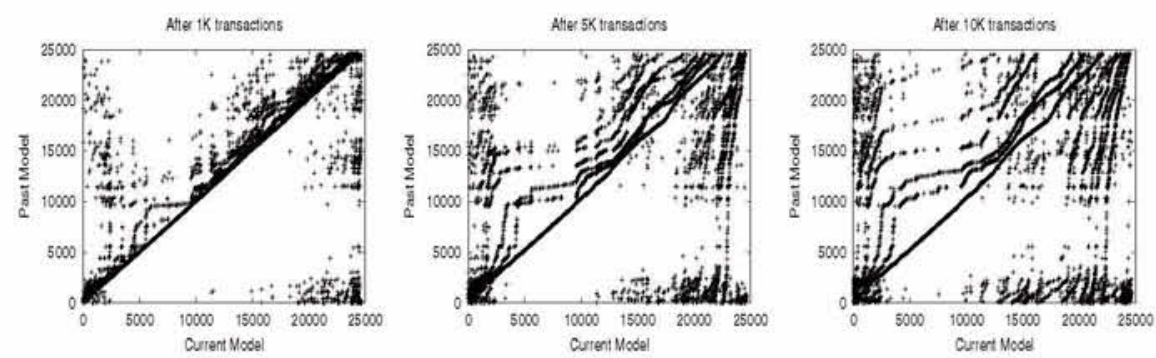

Fig. 1. Evolution of Frequent Itemsets. The X-Axis represents a Totally Accurate Ranking, while the Y-Axis represents an out-dated Ranking

There are many techniques that can be used to estimate the support of a given itemset. The search for such tools is probably endless, and is out of scope of this paper. We believe that the use of costly (time-wise) and sophisticated tools is unlikely to be useful, since their cost may approach or surpass the cost of executing an exact incremental mining algorithm such as ZIGZAG.

Using simple prediction tools (discussed later in this section) one can classify the set of all frequent itemsets across these three categories. Table 1 depicts the percentage of itemsets in each category for the WCup and WPortal databases as an illustration of the approximate approach's potential. From this table we can 
Table 1. Ratio between Invariant, Predictable and Unpredictable Itemsets

\begin{tabular}{|c|c|c|c|}
\hline Database & Invariant & Predictable & Unpredictable \\
\hline \hline WCup & $7.2 \%$ & $45.3 \%$ & $47.5 \%$ \\
\hline WPortal & $9.1 \%$ & $52.1 \%$ & $38.8 \%$ \\
\hline
\end{tabular}

see that both databases present a significant number of invariant and predictable itemsets.

Note that there exists a major difference between invariant and predictable itemsets. If there is a large number of invariant itemsets in the database, the set of popular itemsets generated will remain accurate for a long time. On the other hand, if there is a large number of predictable itemsets, the model will lose accuracy over time. However, using simple models we show that one can generate pretty good estimates of these predictable itemsets, potentially maintaining the accuracy of the support of the popular itemsets.

WAVE is comprised of two phases. The first phase uses the tidlists associated with 1-items whose union is the itemset whose support we want to estimate. The second phase analyzes the sampled results to determine whether it is necessary to count the actual support of the itemset. Each of these phases is described below.

Phase 1: Discretized Support Estimation - The starting point of Phase 1 is the tidlists associated with 1-itemsets, which are always up-to-date since they are simply augmented by novel transactions. Formally, given two tidlists $l_{\alpha}$ and $l_{\beta}$ associated with the itemsets $\alpha$ and $\beta$, we define that the exact tidlist of $\alpha \cup \beta$ is $l_{\alpha \cup \beta}=l_{\alpha} \cap l_{\beta}$. We estimate the upper bound on the merge of two tidlists as follows. We divide the tidlists into $n$ bins. The upper bound of the intersection of corresponding bins is the smallest of the two bin values (each bin value corresponding to the number of entries in the bin). Note, that as long as transactions are ordered temporally, each bin gives us an approximate idea as to how a particular itemset behaved during a given time frame. The upper bounds associated with the bins are then used as input to our estimation technique, described next.

Phase 2: Support Estimation based on Linear Trend Detection - Phase 2 takes as input the information provided by Phase 1 in order to detect trends in itemset frequency. Trend detection is a valuable tool to predict the frequent itemsets behavior in the context of evolving databases. One of the most widespread trend detection techniques is linear regression, that finds a straight line that more closely describes the dataset. The model used by the linear regression is expressed as the function $y=a+b x$, where $a$ is the $y$-intercept and $b$ is the slope of the line that represents the linear relationship between $x$ and $y$. In our scenario the $x$ variable represents the number of transactions while the $y$ variable represents the estimated support (obtained as a function of the upper bound estimates 
from Phase 1). The method of least squares determines the values of $a$ and $b$ that minimize the sum of the squares of the errors, and it is widely used for generating linear regression models.

To verify the goodness of the model generated by the linear regression, we use the $R^{2}$ metric (which takes on values in the range 0 to 1 ) that reveals how closely the estimated $y$-values correlate to its actual $y$-values. A $R^{2}$ value close to 1 indicates that the regression equation is very reliable. In such cases, WAVE provides an approximated technique to find the support of predictable itemsets, an approach that does not have an analog in the itemset mining research. Whenever an itemset is predictable, its support can be simply predicted using the linear regression model, rather than recomputed with expensive database scans. Figure 2 shows the $R^{2}$ distribution for the two databases used in the experiments. This estimate technique achieves extraordinary savings in computational and I/O requirements, as we will see in Section 4.
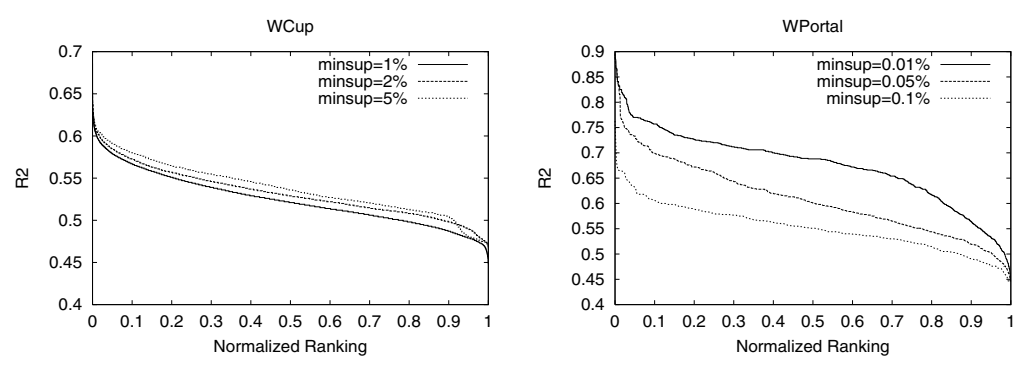

Fig. 2. $R^{2}$ Distribution in WCup and WPortal Databases

\section{Experimental Evaluation}

In this section we evaluate the precision, performance and scalability of WAVE and compare it to other incremental approaches. Real databases from actual applications were used as inputs in the experiments. The first database, WCup, comes from click stream data from the official site of the 1998 World Soccer Cup. WCup was extracted from a 62-day log, comprising 2,128,932 transactions over 4,768 unique items with an average transaction length of 8.5 items and a standard deviation of 11.2. The second database represents the access patterns of a Web Portal. The database, WPortal, comprises 432,601 transactions over 1,182 unique items, and each transaction contains an average length of 2.9 items. Our evaluation is based on three parameters given to WAVE:

Approximation tolerance $-R^{2}$ : the maximum approximation error acceptable. 
Longevity: the number of transactions added to the database which triggers a complete update process.

Base length: the number of transactions effectively mined before we start the estimating process.

Thus, for each minimum support used, we performed multiple executions of the algorithm in different databases, where each execution employs a different combination of $R^{2}$, longevity, and base length. Further, we employed three metrics in our evaluation:

Precision: This metric quantifies how good the approximation is. It is the linear correlation of two ordered sets of itemsets. The ranking criteria is the support, that is, two ordered sets are totally correlated if they are of the same length, and the same itemset appears in corresponding positions in both sets.

Work: This metric quantifies the amount of work performed by WAVE when compared to ULI. We measure the elapsed time for each algorithm while mining a given database in a dedicated single-processor machine. We then calculate the work as the ratio between the elapsed time for our approach and the elapsed time for ULI.

Resource consumption: This metric quantifies the amount of memory used by each algorithm. Observing this metric is interesting for the sake of practical evaluation of the use of WAVE in large databases.

The experiments were run on an IBM - NetFinity $750 \mathrm{MHz}$ processor with $512 \mathrm{MB}$ main memory. The source code for ULI [15], the state-of-the-art algorithm which was used to perform our comparisons, was kindly provided to us by its authors. Timings used to calculate the work metric are based on wall clock time.

\subsection{Accuracy Experiments}

Here we report the accuracy results for the databases described above. Firstly, we evaluate the precision achieved by WAVE. Next, we evaluate the gains in precision provided by WAVE. We employed different databases, minimum supports, base lengths, longevities, and $R^{2}$. Figure 3(a) depicts the precision achieved by WAVE in the WCup database. From this figure we can observe that, as expected, the precision increases with the $R^{2}$ used. Surprisingly, for this database the precision decreases with the base length used. Further, the precision decreases with both the longevity and minimum support.

Slightly different results were observed for the same experiment using the WPortal database. As expected the precision decreases with the longevity. For base lengths as small as $50 \mathrm{~K}$ transactions the lowest precision was achieved by the largest minimum support. We believe that this is because these small base lengths do not provide sufficient information about the database. For base lengths as large as $100 \mathrm{~K}$ transactions, the lowest precision was always achieved by the lowest 
minimum support. Interestingly, the highest precision was initially provided by the highest minimum support, but as we increase the $R^{2}$ value we notice a crossover point after which the second largest support value was the most precise.

We also evaluate the gains in precision achieved by WAVE. From Figure 4(a) we can observe that, using the WCup database, WAVE provides larger gains in precision for smaller values of minimum support. The opposite trend is observed when we evaluate the precision varying the longevity, that is, in general larger gains are achieved by larger longevities. It is obvious that WAVE loses precision over the time, but this result shows that WAVE can maintain a more accurate picture of the frequent itemsets for more time. Finally, the precision increases with the $R^{2}$ value, that is, increasing the precision criteria results in improved prediction precision.

The gains in precision achieved by WAVE were also evaluated using the WPortal database, and the results are depicted in Figure 4(b). In general we observe large gains for smaller values of minimum support. We can also observe that, in all cases, the higher the value of longevity, the larger is the gain in precision. One more time WAVE shows to be very robust in preserving the precision.
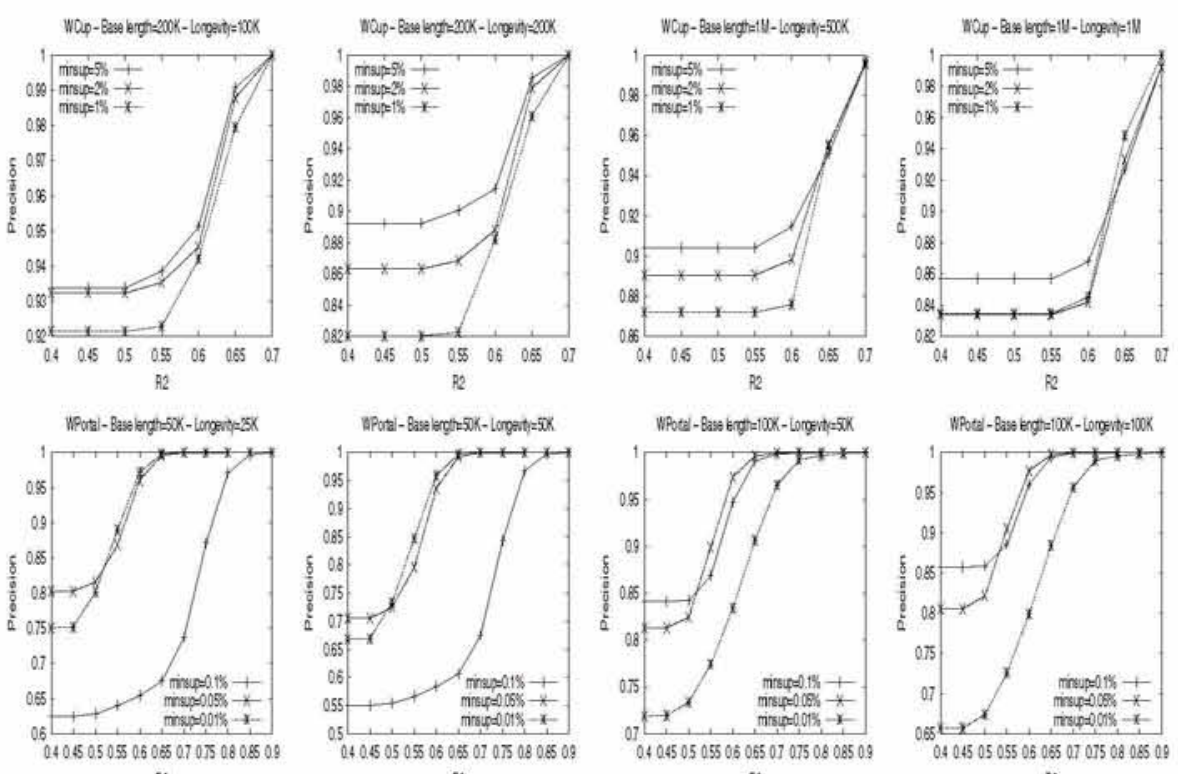

Fig. 3. Precision achieved by WAVE when varying minimum support, $R^{2}$, base length, and longevity for a) WCup Database (top row), and b) WPortal Database (bottom row) 

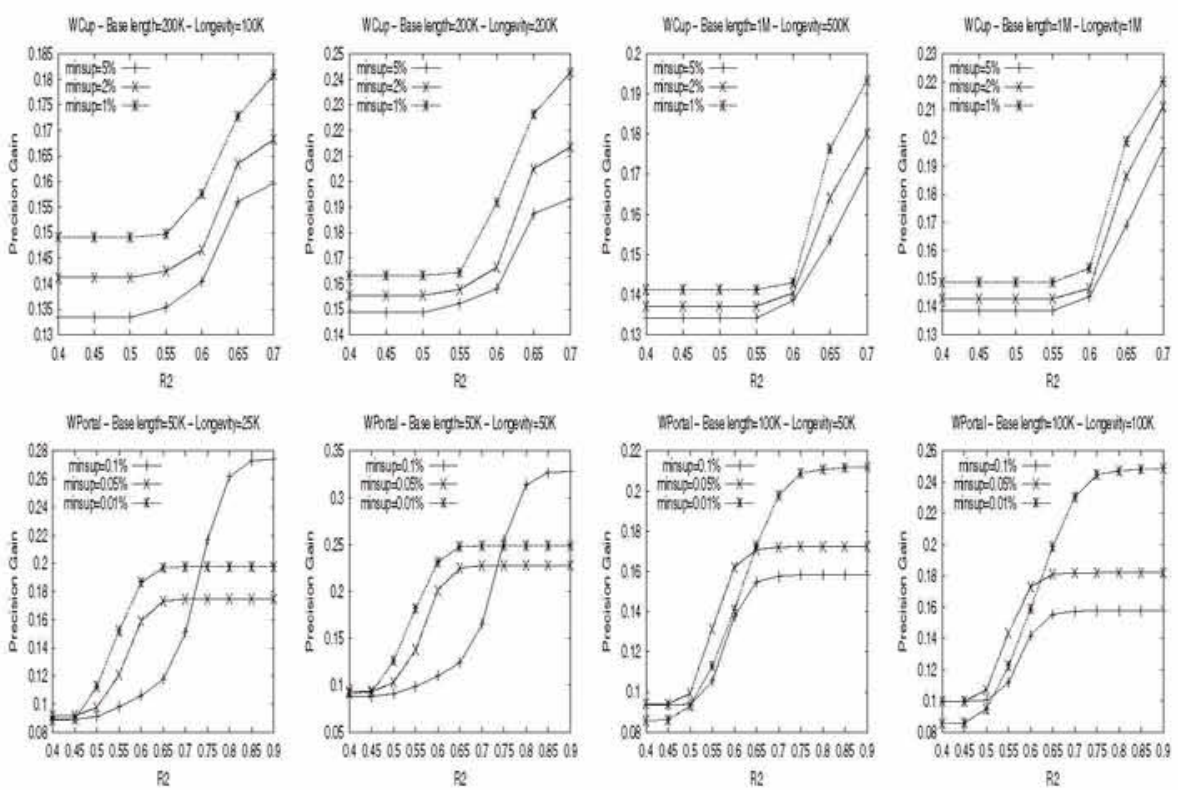

Fig. 4. Precision Gains provided by WAVE when varying minimum support, $R^{2}$, base length, and longevity for a) WCup Database (top row), and b) WPortal Database (bottom row)

\subsection{Performance Experiments}

Now we verify the amount of work performed by WAVE in order to generate an approximate model of associations. From Figure 5(a) we can observe the results obtained using the WCup database. WAVE performs less work for smaller values of minimum support. This is mainly because ULI spent much more time than WAVE in mining with smaller values of minimum support. We can also observe that WAVE performs the same amount of work when the $R^{2}$ threshold reaches the value 0.7 , no matter how much the minimum support value is. The reason is that there are only few itemsets with an approximation as good as 0.7 , and all these itemsets have a support higher than $5 \%$, which was the highest minimum support used in this experiment.

We also verify the performance of WAVE using the WPortal database. In Figure 5(b) we can observe that in general, for this database, WAVE performs less work for smaller values of minimum support. This trend was observed when the database has a size of $50 \mathrm{~K}$ transactions, but an interesting result arises for databases with larger sizes as $100 \mathrm{~K}$ transactions. For smaller values of $R^{2}$, WAVE performs less work for larger values of minimum support, but when we increase the value of $R^{2}$, WAVE performs less work for smaller values of minimum support. The reason is that when the minimum support is too small, a great 
number of itemsets present a poor estimate. When the $R^{2}$ value is also small, even these poor estimates (not so poor as the $R^{2}$ value) are performed. However the relative number of estimates and candidates generated is higher for higher values of minimum support, and, as a consequence, more estimates were performed for higher values of minimum supports. For this database, in all cases, the larger the longevity, the smaller is the work performed by WAVE. Finally, as we can observe in this figure, WAVE performs less work for larger databases.
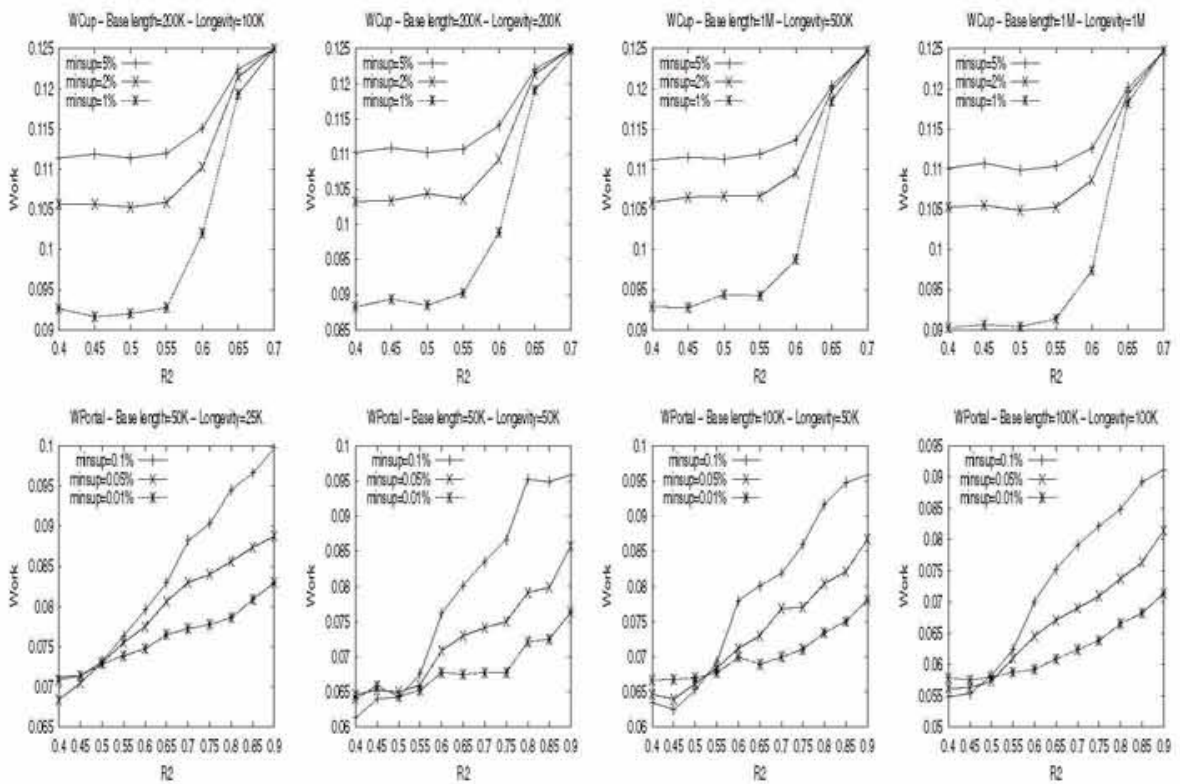

Fig. 5. Work Performed by WAVE when varying minimum support, $R^{2}$, base length, and longevity for a) WCup Database (top row), and b) WPortal Database (bottom row)

\subsection{Scalability Experiments}

In this section we compare the amount of memory used by WAVE and ULI, when we employ different databases, minimum supports, base lengths, longevities, and $R^{2}$. Note that the amount of memory used by ULI does not depend on the $R^{2}$ employed. From Figure 6(a), where we plot the relative amount of memory used by WAVE and ULI to mine the WCup database, we can observe that in all cases WAVE uses less memory than ULI. The amount of memory used by WAVE exponentially decreases with the $R^{2}$ used. This result was expected since for smaller values of $R^{2}$ a larger number of estimates are performed. When we 
decrease the minimum support value, the relative use of memory also decreases. This is because WAVE is more scalable than ULI, with respect to memory usage. The relative memory usage is smaller when we employ larger longevities. Finally, the larger the base length used, the less relative memory usage is observed. As can be seen in Figure 6(b), similar results were observed when we used the WPortal database.
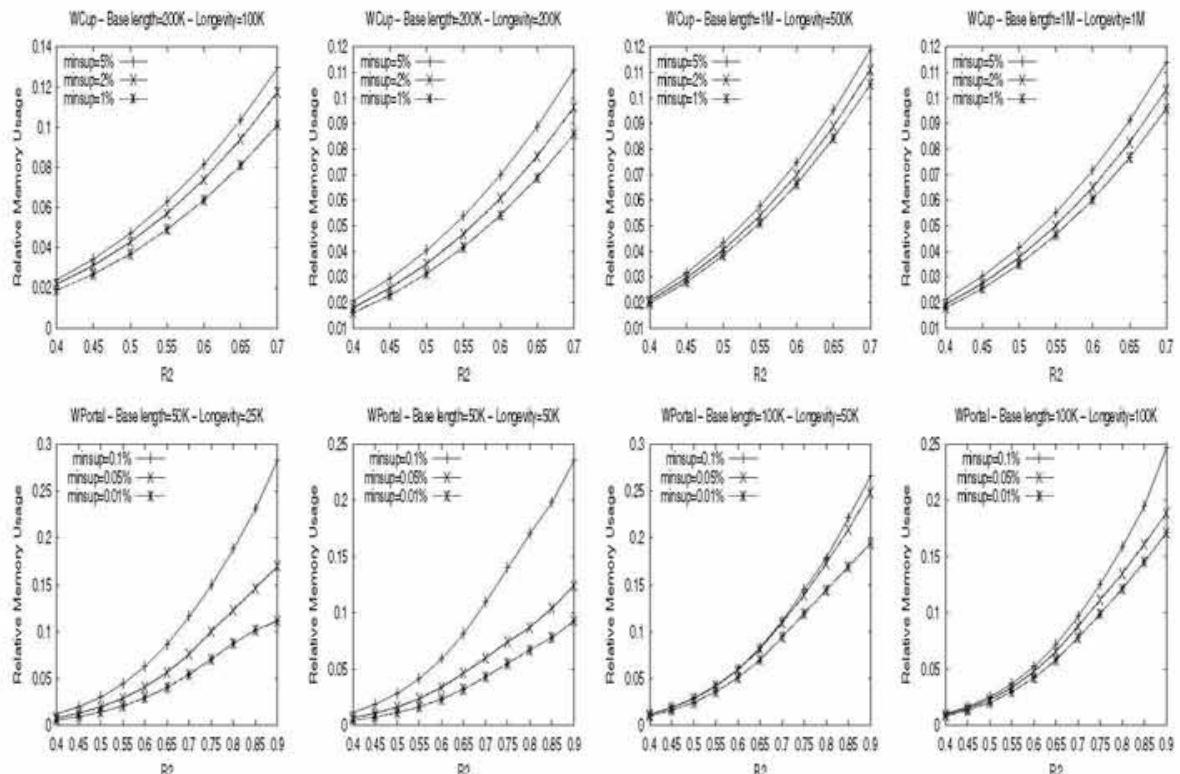

Fig. 6. Relative Memory Usage when varying minimum support, $R^{2}$, base length, and longevity for a) WCup Database (top row), and b) WPortal Database (bottom row)

\section{Conclusions and Future Work}

This paper introduced WAVE, an algorithm capable of generating highly accurate approximate models of associations in evolving databases. WAVE is able to efficiently maintain the model of associations up-to-date within a tolerance threshold value. The resulting accuracy is similar to what would be obtained by reapplying any conventional association mining algorithm to the entire database. Extensive empirical studies on real and synthetic datasets show that WAVE yields very accurate models while at the same time being space and time efficient.

We plan to apply WAVE to more real-world problems; its ability to do selective updates should allow it to perform very well on a broad range of tasks. 
Currently WAVE incrementally maintains the information about the previously frequent itemsets and discards the other ones, but in some domains these recently infrequent itemsets may become useful down the line - identifying such situations based on trend detection and taking advantage of them is another interesting direction for future work.

\section{References}

1. R. Agrawal and R. Srikant. Fast algorithms for mining association rules. In Proc. of the 20 ${ }^{\text {th }}$ Int'l Conf. on Very Large Databases, SanTiago, Chile, June 1994. 436

2. D. Cheung, J. Han, V. Ng, and C. Y. Wong. Maintenance of discovered association rules in large databases: An incremental updating technique. In Proc. of the $12^{\text {th }}$ Intl. Conf. on Data Engineering, February 1996. 436, 437, 438

3. D. Cheung, K. Hu, and S. Xia. Asynchronous parallel algorithm for mining association rules on a shared-memory multipprocessors. In ACM Symposium on Parallel Algorithms and Architectures, pages 279-288, 1998. 436

4. D. Cheung, S. Lee, and B. Kao. A general incremental technique for maintaining discovered association rules. In Proc. of the $5^{\text {th }}$ Intl. Conf. on Database Systems for Advanced Applications, pages 1-4, April 1997. 436, 437, 438

5. V. Ganti, J. Gehrke, and R. Ramakrishnan. Demon: Mining and monitoring evolving data. In Proc. of the $16^{\text {th }}$ Int'l Conf. on Data Engineering, pages 439-448, San Diego, USA, May 2000. 437, 438

6. K. Gouda and M. Zaki. Efficiently mining maximal frequent itemsets. In Proc. of the $1^{\text {st }}$ IEEE Int'l Conference on Data Mining, San Jose, USA, November 2001. 436,437

7. J. Han, H. Jamil, Y. Lu, L. Chen, Y. Liao, and J. Pei. Dna-miner: A system prototype for mining dna sequences. In Proc. of the 2001 ACM-SIGMOD Int'l. Conf. on Management of Data, Santa Barbara, CA, May 2001. 436

8. J. Han, J. Pei, and Y. Yin. Mining frequent patterns without candidate generation. In Proc. of the ACM SIGMOD Int'l Conf. on Management of Data, May 2000. 436

9. C. Kamath. On mining scientific datasets. In et al R. L. Grossman, editor, Data Mining for Scientific and Engineering Applications, pages 1-21. Kluwer Academic Publishers, 2001. 436

10. S. Lee and D. Cheung. Maintenance of discovered association rules: When to update? In Research Issues on Data Mining and Knowledge Discovery, page March, 1997. 437

11. H. Mannila and H. Toivonen. Levelwise search and borders of theories in knowledge discovery. In Technical Report TR C-1997-8, U. of Helsinki, January 1997. 437

12. S. Parthasarathy, M. Zaki, M. Ogihara, and S. Dwarkadas. Incremental and interactive sequence mining. ACM Confereince on Information and Knowledge Management (CIKM), Mar 1999. 437

13. S. Parthasarathy, M. Zaki, M. Ogihara, and W. Li. Parallel data mining for association rules on shared-memory systems. In Knowledge and Information Systems, Santa Barbara, CA, February 2001. 436

14. M. Rajman and R. Besan. Text mining - knowledge extraction from unstructured textual data. In Proc. of the $6^{\text {th }}$ Int'l Conf. Federation of Classication Societies, pages 473-480, Roma, Italy, 1998. 436

15. S. Thomas, S. Bodagala, K. Alsabti, and S. Ranka. An efficient algorithm for the incremental updation of association rules. In Proc. of the $3^{\text {rd }}$ Int'l Conf. on Knowledge Discovery and Data Mining, August 1997. 436, 437, 438, 442 
16. A. Veloso, W. Meira Jr., M. B. de Carvalho, B. Pôssas, S. Parthasarathy, and M. Zaki. Mining frequent itemsets in evolving databases. In Proc. of the $2^{\text {nd }}$ SIAM Int'l Conf. on Data Mining, Arlington, USA, May 2002. 436, 437, 438

17. A. Veloso, B. Rocha, W. Meira Jr., and M. de Carvalho. Real world association rule mining. In Proc. of the 19th British National Conf. on Databases (to appear), July 2002. 437

18. M. Zaki and C. Hsiao. Charm: An efficient algorithm for closed itemset mining. In Proc. of the $2^{\text {nd }}$ SIAM Int'l Conf. on Data Mining, Arlington, USA, May 2002. 436, 437

19. M. Zaki, S. Parthasarathy, M. Ogihara, and W. Li. New algorithms for fast discovery of association rules. In Proc. of $3^{\text {rd }}$ Int'l Conf. Knowledge Discovery and Data Mining, August 1997. 436

20. M. Zaki, S. Parthasarathy, M. Ogihara, and W. Li. New parallel algorithms for fast discovery of association rules. Data Mining and Knowledge Discovery: An International Journal, 4(1):343-373, December 1997. 436 